\title{
Bovine coronary region keratinocyte colony formation is supported by epidermal-dermal interactions
}

\author{
J. A. Mills, ${ }^{*}$ D. S. Zarlenga, $\dagger$ and R. M. Dyer ${ }^{1}$ \\ *Thomas Jefferson University Hospital, Philadelphia, PA 19107 \\ †Bovine Functional Genomics Laboratory and Animal Parasitic Diseases Laboratory and US National Parasite Collection, \\ US Department of Agriculture, Beltsville, MD 20705 \\ $\ddagger$ Department of Animal and Food Sciences, College of Agriculture and Natural Resources, University of Delaware, Newark 19717
}

\section{ABSTRACT}

Delineating the factors that orchestrate keratinocyte growth and differentiation in the claw is pivotal to understanding the quality of hoof horn production in health and disease. The specific objectives of this investigation were to establish an in vitro culture system for bovine coronary region keratinocytes and dermal fibroblasts, determine the colony-forming capacity of epidermal keratinocytes in the coronary region, and characterize transcriptional changes in specific cytokine, growth factor, and receptor genes during colony formation in coculture. Fibroblasts and keratinocytes from the coronary region of the lateral, hind limb claw were collected, and $5.0 \times 10^{3}$ and $7.5 \times 10^{3}$ keratinocytes were cultured in the presence or absence of fibroblast monolayers, respectively. The 2 densities of keratinocytes formed $144 \pm 15.8$ and $183 \pm 26.9$ colonies, respectively, in the presence of dermal fibroblasts, whereas no colonies developed in the absence of dermal fibroblasts. Keratinocytes with the ability to show colony formation comprised $1.09 \% \pm 0.16$ to $1.77 \% \pm$ 0.28 of the keratinocyte population isolated from the coronary region. Keratinocyte-fibroblast cocultures developed a time-dependent increased expression of several growth factors, cytokines, and receptors. These findings demonstrated that keratinocytes from the bovine coronary region formed colonies in vitro and that colony formation occurred with an absolute dependence on dermal fibroblasts. Colony growth was associated with increased transcriptional expression of cytokine, growth factor, and receptor expression known to drive keratinocyte colony formation in other species. The results indicate that horn-producing keratinocytes must interact with dermal fibroblasts during normal tissue homeostasis in the bovine claw.

Key words: keratinocyte, coronary region, growth factor, dermis

Received June 3, 2008.

Accepted December 13, 2008.

${ }^{1}$ Corresponding author: rdyer@udel.edu

\section{INTRODUCTION}

Lesions of the hind limb claw horn are the dominant cause of bovine lameness (Murray et al., 1996). Poorquality horn tissue associated with a loss of elasticity and hardness has been proposed to underlie claw horn lesions. Biochemical, mechanical, and morphologicanatomic data associated poor-quality horn tissue with failure of microfilament cross-linking, defective envelope cornification, and changes in lipid composition of the intercellular cement-like substance between keratinocytes (Mulling et al., 1999). Normal bovine hoof horn tissue develops through a process of intracellular keratinization, microfilament synthesis and cross-linking, protein and lipid deposition around the plasma membrane, and finally cross-linking of envelope proteins (Tomlinson et al., 2004). A program of terminal differentiation orchestrates these processes as squamous epithelial cells progress through the stratified tissue layers. Mitotically active cells with self-renewal properties in the innermost basal layer generate progeny that exit the basal cell niche. Terminally differentiating progeny migrate through overlying suprabasal layers to replace desquamated cornified cells shed from the outermost layers.

The lack of knowledge about biologic events orchestrating normal growth, differentiation, and maturation of claw horn tissue creates challenges for understanding claw horn production during periods of health and disease. In murine and human integuments, cell-to-cell communications between dermal fibroblasts and epidermal keratinocytes direct epithelial growth and differentiation in the epidermis (Smola et al., 1993; MaasSzabowski et al., 1999; Werner and Smola, 2001). Although keratinocytes are separated from the underlying dermal fibroblasts by a basement membrane, the normal homeostatic balance between keratinocyte growth and differentiation is maintained through keratinocyte-dermal fibroblast interactions. Disease-induced perturbations in homeostasis could lead to defective claw horn production. This model provides a framework in which epidermal-dermal interactions governing bovine claw horn production can be explored. The seminal studies 
of Rheinwald and Green (1975) established techniques for keratinocyte propagation to determine the colonyforming activity and clonal activity in populations of keratinocytes. From that point, a body of work on keratinocyte proliferation and differentiation has led to a better understanding of keratinocyte behavior under normal and pathologic conditions.

Factors regulating keratinocyte growth and development can be evaluated in cell culture models. To that end, we established an in vitro culture system of bovine coronary region keratinocytes and utilized this system to address 3 questions: 1) Do progenitor cells capable of keratinocyte colony formation exist within keratinocyte populations recovered from the bovine coronary region? 2) Are fibroblasts required to support keratinocyte growth and colony formation? 3) Are cell-to-cell interactions during keratinocyte growth and colony formation accompanied by changes in the expression of several cytokine and growth factor genes normally associated with keratinocyte colony growth in murine and human models?

\section{MATERIALS AND METHODS}

\section{Histology and Immunohistochemistry}

Histological analysis was performed in tissues excised from the epidermis and dermis of a normal, left, lateral hind claw of 6 mature, lactating Holstein Friesian cows. A tissue sample approximately $1 \times 1 \mathrm{~cm}$ of epidermis and dermis from the lateral coronary region, lateral wall, sole, and bulb region of the lateral claw was removed, placed in $10 \%$ buffered formalin for $24 \mathrm{~h}$, and then embedded in paraffin. The paraffin-embedded specimens were cut into $4-\mu \mathrm{m}$ sections, placed on silane-coated glass slides, and stained with hematoxylin and eosin for histological examination.

Immunohistochemical studies were performed using the paraffin-embedded sections of the coronary region described previously. Sections were heated $\left(56^{\circ} \mathrm{C}\right)$ for 25 min, deparaffinized in $100 \%$ xylene, serially passed through graded ethanol (100, 90, and $80 \%)$ and rehydrated in distilled water. For antigen recovery, samples were placed in $10 \mathrm{~m} M$ sodium citrate with $0.1 \%$ Tween-20, and heated at $100^{\circ} \mathrm{C}$ under pressure $(35$ min). After washing in Hanks' buffered saline solution (HBSS), sections were blocked with $10 \%$ goat serum in 0.1\% Tween-20 in HBSS for $30 \mathrm{~min}$ and then incubated for $1 \mathrm{~h}\left(27^{\circ} \mathrm{C}\right)$ with anti-murine p63 (1:200; BD Biosciences, Franklin Lakes, NJ) in 10\% fetal bovine serum (FBS) and HBSS. Sections were washed in HBSS and incubated $1 \mathrm{~h}\left(27^{\circ} \mathrm{C}\right)$ with fluorescein isothiocyanate (FITC)-conjugated goat anti-murine antibody (1:200; Sigma, St. Louis, MO). Appropriate background stain- ing controls were performed (primary antibody alone, secondary antibody alone). Sections were washed 3 times with HBSS, placed under a coverslip with Vectashield (Vector Laboratories Inc., Burlington, CA), and sealed. Immunostained tissue sections were viewed under a fluorescent microscope.

\section{Tissue Extraction and Cell Isolation}

Normal lateral left hind claws $(\mathrm{n}=55)$ obtained from freshly killed, lactating cattle were scraped, trimmed, and washed free of feces. Abaxial surfaces were scrubbed for 10 min and swabbed with betadine solution (Betadine, VetCo, St. Joseph, MO) and isopropyl alcohol. Two cuts, $5.0 \mathrm{~cm}$ apart, were placed in the lateral wall extending from the coronary region toward the distal wall approximately 4.0 to $5.0 \mathrm{~cm}$. The cuts were connected distally and the section of wall removed by traction. Sections $(4.0 \mathrm{~cm}$ long $\times 1.0$ $\mathrm{cm}$ wide) of the underlying coronary region epidermis and dermis were aseptically removed, transferred into $1.5 \%$ trypsin (Amresco, Solon, $\mathrm{OH}$ ) in divalent cationfree HBSS $\left(25^{\circ} \mathrm{C}\right)$ and incubated for $2 \mathrm{~h}$ at $37^{\circ} \mathrm{C}$. The tissues were minced and scraped into suspensions and fine pieces before suspension in Dulbecco's Minimum Essential Media (DMEM; Biosource, Rockville, MD) supplemented with $10 \%$ FBS, $100 \mathrm{U} / \mathrm{mL}$ penicillin, 100 $\mu \mathrm{g} / \mathrm{mL}$ streptomycin, and $2 \mathrm{~m} M$ glutamine, and then overlaid onto 70 to $85 \%$ confluent bovine dermal fibroblast monolayers. In some cases, primary keratinocyte suspensions employed in the colony-forming unit assays were prepared from scraped and minced tissues by filtration through wire mesh or sterile gauze to remove tissue debris before cell enumeration and assay.

Minced tissues were propagated 14 to $21 \mathrm{~d}$ in DMEM supplemented with $10 \%$ FBS, $100 \mathrm{U} / \mathrm{mL}$ penicillin, 100 $\mu \mathrm{g} / \mathrm{mL}$ streptomycin, and $2 \mathrm{~m} M$ glutamine with media changes every $4 \mathrm{~d}$. These fibroblast-keratinocyte cocultures were separated into fibroblast- or keratinocyteenriched fractions by incubation in $0.7 \mu M$ EDTA in divalent-free HBSS for 2 min $\left(25^{\circ} \mathrm{C}\right)$ followed by washing $(3 \times)$ in $\mathrm{Ca}-/ \mathrm{Mg}$-free HBSS. Fibroblast-enriched supernatants were collected, washed $3 \times$ in HBSS, and recultured in DMEM, 10\% FBS, penicillin, $100 \mu \mathrm{g} / \mathrm{mL}$ streptomycin, and $2 \mathrm{mM}$ glutamine. Adherent keratinocyte fractions were placed in keratinocyte growth medium containing $0.2 \mathrm{ng} / \mathrm{mL}$ epidermal growth factor and $0.25 \mu \mathrm{g} / \mathrm{mL}$ bovine pituitary extract (Invitrogen Inc., Grand Island, NY) for 7 d. Fibroblast and keratinocytes were characterized with the fibroblast marker vimentin using Cy3-conjugated murine anti-vimentin (Sigma). Cell monolayers were washed (HBSS), fixed (10 $\mathrm{min})$ in $3.7 \%$ paraformaldehyde, and blocked with $0.1 \%$ Triton X-100 and 10\% FBS (30 min). Cell prepa- 
Table 1. Primers used for real-time PCR amplification and primer efficiency for each gene

\begin{tabular}{|c|c|c|c|c|}
\hline Identification $^{1}$ & Polarity & Primer sequence $\left(5^{\prime}-3^{\prime}\right)$ & Length, bp & Efficiency \\
\hline \multirow[t]{2}{*}{$\mathrm{IL}-1 \alpha$} & Forward & CACTTCGTGAGGACCAGATGAA & 22 & \multirow[t]{2}{*}{1.93} \\
\hline & Reverse & TGCCACCATCACCACATTCT & 20 & \\
\hline IL-1 $\beta$ & Reverse & GAGAGGGTTTCCATTCTGAAGTCA & 24 & 1.88 \\
\hline \multirow[t]{2}{*}{ TNF $\alpha$} & Forward & CGGTGGTGGGACTCGTATG & 19 & \multirow[t]{2}{*}{1.70} \\
\hline & Reverse & GCTGGTTGTCTTCCAGCTTCA & 21 & \\
\hline & Reverse & GGGCTGGAACAGTTCACATT & 20 & 1.86 \\
\hline \multirow[t]{2}{*}{ TGF $\beta$} & Forward & GAACTGCTGTGTTCGTCAGC & 20 & \multirow[t]{2}{*}{1.80} \\
\hline & Reverse & TCCAGGCTCCAGATGTAAGG & 20 & \\
\hline \multirow[t]{2}{*}{ GM-CSF } & Forward & CAGCTTCTCCGCACCTACTC & 20 & \multirow[t]{2}{*}{1.98} \\
\hline & Reverse & TTGGTTCCTGGGAGCAAC & 18 & \\
\hline \multicolumn{5}{|l|}{ Receptors } \\
\hline KGF receptor IIIb & Forward & AGCAAATGCCTCCACTGTGG & 20 & 1.73 \\
\hline \multirow[t]{2}{*}{ IL-1 receptor antagonist } & Forward & AGATGCCTGTCCACTCTGCT & 20 & \multirow[t]{2}{*}{1.89} \\
\hline & Reverse & TGGAGACTGGGTCTTGTTCC & 20 & \\
\hline \multicolumn{5}{|l|}{ Housekeeping genes } \\
\hline Ubiquitin-C & Forward & TTCAGTCTTCGTTCTTCTGTGT & 22 & \multirow[t]{2}{*}{1.94} \\
\hline & Reverse & CAAAGTGATGGTTTTACCAGT & 21 & \\
\hline
\end{tabular}

${ }^{1} \mathrm{TNF} \alpha=$ tumor necrosis factor $\alpha$ KGF $=$ keratinocyte growth factor; TGF $\beta=$ transforming growth factor $\beta$; GM-CSF $=$ granulocyte colony stimulating factor.

rations were incubated with guinea pig anti-fetal bovine hoof keratin (1:20; Sigma) or Cy3-conjugated murine anti-vimentin (1:400 dilution; $\left.1 \mathrm{~h}, 4^{\circ} \mathrm{C}\right)$. Monolayers reacted with anti-bovine fetal hoof keratin were washed $3 \times$ in PBS and incubated with FITC-conjugated goat anti-guinea pig IgG (1:200; Sigma) for $30 \mathrm{~min}$ at $37^{\circ} \mathrm{C}$. Then, all monolayers were washed $3 \times$ in PBS and fixed with Vectorshield $(15 \mu \mathrm{L})$.

Cell viability was determined with $4 \%$ trypan blue dye exclusion. Keratinocyte viability, numbers, and purity were $77.0 \% \pm 4.1,9.3 \times 10^{5} \pm 1.5 \times 10^{5}$, and $96.0 \% \pm 2.0$, respectively $(\mathrm{n}=8)$.

\section{Determination of Colony-Forming Units}

Fibroblasts were plated at $1.0 \times 10^{4}$ cells $/ \mathrm{cm}^{2}$ in DMEM with $10 \% \mathrm{FBS}, 100 \mathrm{U} / \mathrm{mL}$ penicillin, $100 \mu \mathrm{g} /$ $\mathrm{mL}$ streptomycin, and $2 \mathrm{~m} M$ glutamine, grown to confluence, and treated with $12 \mu M$ mitomycin C (2 h) to inhibit fibroblast proliferation. Second-passage keratinocytes in keratinocyte growth medium with $0.2 \mathrm{ng} / \mathrm{mL}$ epidermal growth factor and $0.25 \mu \mathrm{g} / \mathrm{mL}$ bovine pituitary extract were washed once in HBSS, incubated in trypsin $(0.5 \mathrm{~g} / \mathrm{L})$ and $0.5 \mu M$ ethylene glycol tetraacetic acid (EGTA; Sigma Chemical, St. Louis, MO) in $0.9 \% \mathrm{NaCl}$ for $20 \mathrm{~min}$ (Sigma), washed
$3 \times$ in HBSS, and suspended in DMEM with $10 \%$ FBS, penicillin, $100 \mu \mathrm{g} / \mathrm{mL}$ streptomycin, and $2 \mathrm{mM}$ glutamine. To assess formation of colony-forming units, primary or second-passage keratinocytes were seeded at $5.0 \times 10^{3}, 7.5 \times 10^{3}$, and $1.0 \times 10^{4}$ viable cells/ $\mathrm{cm}^{2}$ onto confluent bovine dermal fibroblast monolayers (Table 1) and incubated up to $10 \mathrm{~d}$ in DMEM with $10 \%$ FBS. Cocultures were washed with HBSS $(3 \times)$, fixed in $3.7 \%$ buffered formaldehyde (30 min), and stained with $1 \%$ rhodanile blue (Sigma) for $45 \mathrm{~min}$ in a nonhumidified incubator at $37^{\circ} \mathrm{C}$ (Rheinwald and Green, 1975). Stained cultures were washed $(5 \times)$ with distilled water and evaluated for colony formation. Clusters of at least 3 keratinocytes indicated the presence of a colony.

Colony-forming unit assays were also performed as described but in the presence of $0.1 \%$ FBS instead of $10.0 \%$ FBS. Preliminary experiments determined that fibroblasts grew well in $10.0 \%$ FBS, whereas they remained viable but did not grow in $0.1,0.5$, or $1.0 \%$ FBS (data not shown). This observation established conditions whereby fibroblast requirements for keratinocyte colony formation could be evaluated without growth of contaminating fibroblasts in fibroblast-depleted keratinocyte controls. Accordingly, $5.0 \times 10^{3}$ and $7.5 \times 10^{3}$ viable second-passage keratinocytes were seeded in the absence or presence of dermal fibroblast monolayers in 
DMEM supplemented with $0.1 \%$ FBS. Cell preparations were incubated for $6 \mathrm{~d}$ and stained with rhodanile blue.

\section{Long-Term Gene Expression in Keratinocyte- Fibroblast Coculture}

Cytokine and growth factor mRNA was evaluated in cocultures incubated in 10\% FBS. Second-passage keratinocytes were seeded $\left(7.5 \times 10^{3}\right.$ cell $\left./ \mathrm{cm}^{2}\right)$ onto confluent fibroblast monolayers maintained in $10 \%$ FBS before initiation of the experiments. Cocultures were incubated for $0,12,24,48$, and $144 \mathrm{~h}$ before RNA isolation and real-time PCR. Total RNA was extracted from the tissue and cell cultures using the RNeasy kit plus on-column DNase treatment following the manufacturer's protocol (Qiagen Sciences, Germantown, MD). Total RNA concentrations were determined by UV absorption, and sample integrity was assessed on $1.5 \%$ denaturing agarose gels. Complementary DNA synthesis was performed using Transcriptor cDNA Synthesis Kit (Roche Diagnostics Corp., Indianapolis, IN) according to the manufacturer's recommendations.

\section{Real-Time Reverse Transcription-PCR}

Real-time PCR was performed in an ABI Prism 7900 (Applied Biosystems, Foster City, CA) in 384well optical plates. Each sample was performed in triplicate and ubiquitin $\mathrm{C}$ served as internal control. Complementary DNA was diluted 1:4 and transcripts were determined by real-time PCR using $1 \mu \mathrm{L}(25 \mathrm{ng} /$ sample) of diluted cDNA, $10 \mu \mathrm{L}$ of SYBR green QPCR master mix (Applied Biosystems), and $1 \mu \mathrm{L}(1 \mu M)$ of gene-specific primers (Table 1). Primer efficiency (E) was determined from the slope of standard curves as $\mathrm{E}$ $=-1+10^{(-1 / \text { slope })}$. Universal thermal conditions of 10 min at $95^{\circ} \mathrm{C}$ and 40 cycles of $15 \mathrm{~s}$ at $95^{\circ} \mathrm{C}$ and $1 \mathrm{~min}$ at $60^{\circ} \mathrm{C}$ were utilized. Amplicons were visualized on $1.5 \%$ agarose to verify predicted amplicon size (ranging from 80 to $150 \mathrm{bp}$ depending on the gene). Amplicons were sequenced and melting curves performed to verify presence of a single product with the predicted melting temperature (data not shown).

\section{Statistical Analysis}

Statistical analyses were performed using the GLM (SAS Institute Inc., Cary, NC) for tissue culture experiments with a repeated measures model for colony-forming unit formation and efficiency analysis, Significance was defined as $P<0.05$. Real-time PCR data were normalized using ubiquitin $\mathrm{C}$ as an internal reference gene, and relative expression for each sample was calcu- lated according to Livak and Schmittgen (2001). Data were expressed as relative expression (RE) in arbitrary units determined as $\mathrm{RE}=2^{-}\left[\left(\mathrm{C}_{\mathrm{T}}\right.\right.$ sample $\left._{\mathrm{n}}-\mathrm{C}_{\mathrm{T}} \mathrm{Ubiq}_{\mathrm{n}}\right)$ - $\left(\mathrm{C}_{\mathrm{T}}\right.$ sample $\left.\left._{\text {avg }}-\mathrm{C}_{\mathrm{T}} \mathrm{Ubiq}_{\text {avg }}\right)\right]$, where $\mathrm{C}_{\mathrm{T}}$ was the cutoff threshold of sample or ubiquitin $\mathrm{C}$ fluorescence. Statistical analysis for long-term expression experiments was performed using the MIXED procedure in the SAS system (SAS Institute, 2001) with a repeated measures model for mRNA expression analysis. Significance was defined as $P \leq 0.05$ and a trend was defined as $0.05<$ $P \leq 0.10$.

\section{RESULTS}

\section{Tissue Distribution of p63, an Epithelial Basal Cell Marker}

Histological evaluation was performed on tissues derived from the coronary, wall, sole, and bulb regions. The dermis of all tissues consisted of normal irregular dense fibrous connective tissue, the associated fibroblasts, and vascularity (Figure 1A). Hemorrhage, congestion, thrombosis, atherosclerotic changes, or leukocytic infiltrates were not observed. The basal and overlying epidermal layers appeared normal and were supported by an intact basement membrane. The p63 marker (Figure 1B) stained with a precise nuclear localization within cells restricted to the epidermal layer of the coronary region. The brightest p63 fluorescent signal emanated from the basal layer and, to a lesser degree, the lowest 3 to 4 layers of the stratum spinosum. This distribution was evident only when the plane of the cut was perpendicular (2 examples) or parallel (2 examples) to the long axis of the dermal papillae. As might be expected, oblique and tangential cuts across the papilla obscured this pattern of distribution. The p63 distribution was discernable around papillae cut in longitudinal, oblique, or cross section. The p63 distribution appeared less dense at the apex of the papilla compared with the deep recesses of the interpapilla areas of the coronary region. In addition, areas of strongest p63 signal along the epidermal-dermal junction appeared to be interspersed between areas with weak or even an absence of p63 signal.

\section{Cell Isolation and Culture Characteristics}

During cell extraction, epidermal and dermal portions of the coronary region were minced such that extracts contained variable numbers of free cells and tissue fragments. Upon culture, fibroblasts and keratinocytes migrated from the tissue into the media. Within 3 to $4 \mathrm{~d}$, stellate fibroblasts began to form small, single-cell islets and monolayers dispersed throughout the dish. 

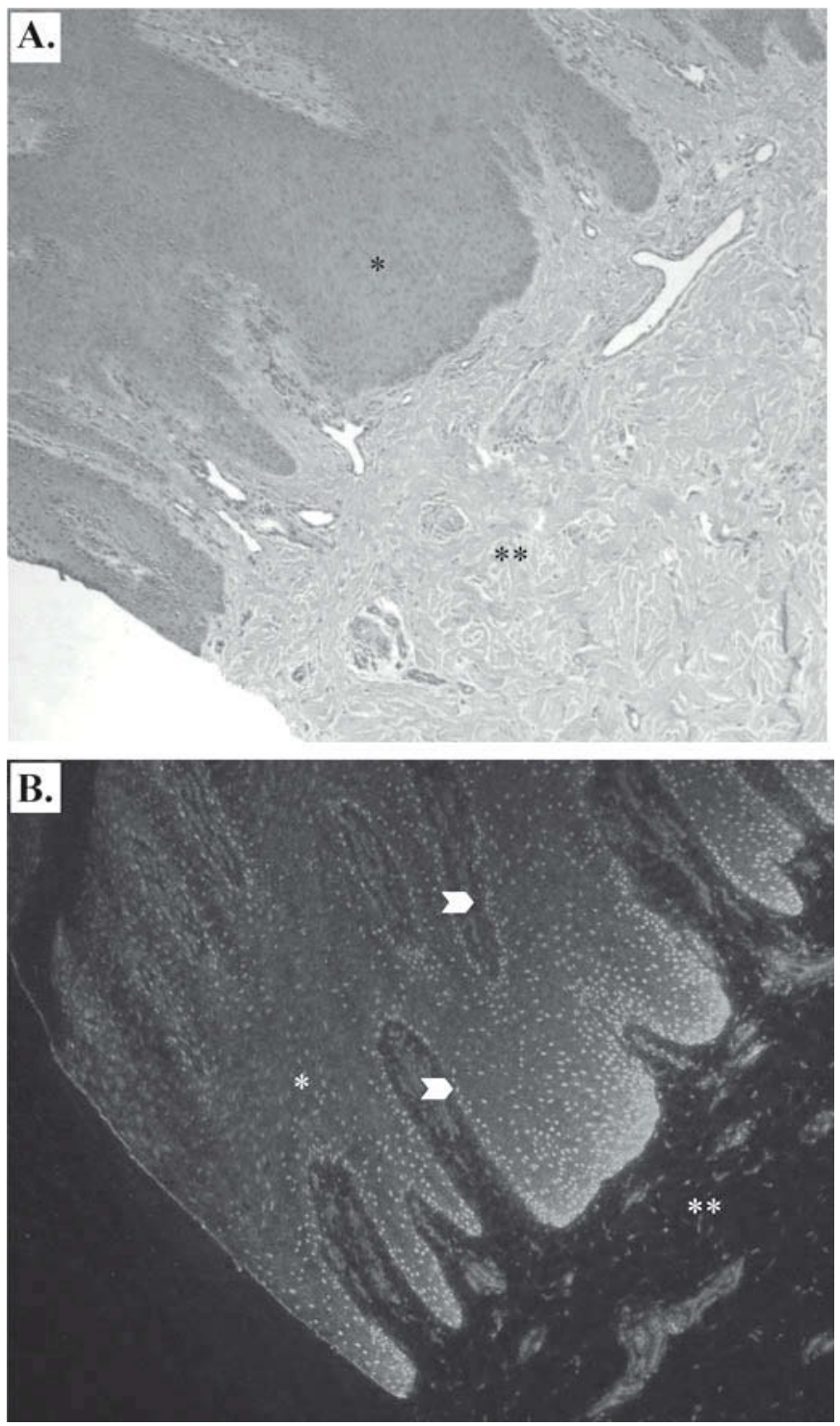

Figure 1. Cross-section of the epidermis and dermal of the bovine coronary region (hematoxylin and eosin-stained; 40× magnification). A) Note the absence of inflammatory cell infiltrates, hemorrhage and lymphocytic infiltrates in the dermal $(* *)$ and overlying epidermal $\left(^{*}\right)$ regions. Immunohistochemical labeling was performed to determine the distribution of the keratinocyte basal cell marker, p63 in the epidermis $\left(^{*}\right)$ and dermis $(* *)$ of the bovine coronary region. Note that p63 distribution is greatest in basal layers at the epidermal-dermal junction in sections where the plane of the section is perpendicular (arrow) or parallel (arrow) to the long axis of the coronary region papilla; B) Picture representative of bovine coronary region epidermis and dermis.

After 7 to $14 \mathrm{~d}$, fibroblasts formed confluent monolayers surrounding individual small, discrete islets of keratinocytes (Figure 2). By 7 d, keratinocytes within islets began to stratify into irregular layers. As islets enlarged, the keratinocytes appeared to push aside the surrounding fibroblasts at the keratinocyte-fibroblast junctions (Figure 2A). Keratinocytes began to form raised colonies and circular outgrowths (Figure 2B) that projected off well-formed, dermal fibroblast monolayers. By d 14 keratinocyte islets varied in size and appeared as opaque plaques with irregular boundaries. Vimentin immunostains of stellate fibroblasts (Figure 3A) produced an uneven, delicate, lace-like distribution of vimentin within the cytoplasm (Figure 3B). Keratin immunostains of the cobblestone keratinocytes in culture (Figure 3C) showed a diffuse, even distribution of keratin throughout the cytoplasm of each cell (Figure 3D). Keratin staining appeared to be unevenly distributed across different keratinocytes within the same colony.

\section{Coronary Region Keratinocytes Formed Colonies in Coculture}

Coronary-region keratinocyte colonies generated by primary or second-passage keratinocytes were readily discernable when stained with $1 \%$ rhodanile blue as the fibroblasts remained unstained or stained faint gray-blue. In contrast, keratinocytes in contact with or proximal to the underlying fibroblasts stained blue, the overlying keratinocytes stained violet, and the most heavily keratinized, superficial keratinocytes stained red. At $2 \mathrm{~d}$, keratinocyte colonies appeared as small clusters of cells associated with the underlying fibroblast monolayer (Figure 4A). Colony size increased by d 6 (Figure $4 \mathrm{~B})$ with stratification of keratinocytes overlying the more basally located keratinocytes. Colonies expanded radially such that by $10 \mathrm{~d}$, the surrounding fibroblasts were displaced to the periphery, and juxtapositioned keratinocyte colonies fused to generate large, irregularly shaped colonies (Figure 4C) with centrally located stacks of irregularly shaped cells. Isolated clusters of cells could be seen within the larger colonies. Time $(P$ $<0.005)$ and keratinocyte input number $(P<0.005)$ affected colony-forming unit numbers in cocultures of either primary (Figure 5A) or second passage (Figure 5B) keratinocytes. Keratinocyte colony numbers in the fibroblast-keratinocyte cocultures containing $5.0 \times 10^{3}$, $7.5 \times 10^{3}$, and $1.0 \times 10^{4}$ primary keratinocytes $/ \mathrm{cm}^{2}$ increased from 2 to $6 \mathrm{~d}$ of coculture (Figure 5A; $P<$ 0.05); however, by $10 \mathrm{~d}$ the number of colony-forming units tended to decrease relative to $6 \mathrm{~d}$ colony numbers but remained greater than $2 \mathrm{~d}$ colony numbers (Figure $5 \mathrm{~A} ; P<0.05)$. Both time in coculture $(P<0.05)$ and keratinocyte number $(P<0.05)$ affected colony formation in second-passage coronary region keratinocytes (Figure 5B). Colony formation in fibroblast-keratinocyte cocultures containing $5 \times 10^{3}, 7.5 \times 10^{3}$, or $1.0 \times 10^{3}$ second-passage keratinocytes $/ \mathrm{cm}^{2}$ increased during the 10-d period (Figure 5B; $P<0.05$ ). Colony formation at $\mathrm{d} 6$ and 10 was greater than that at $\mathrm{d} 2$; however, 


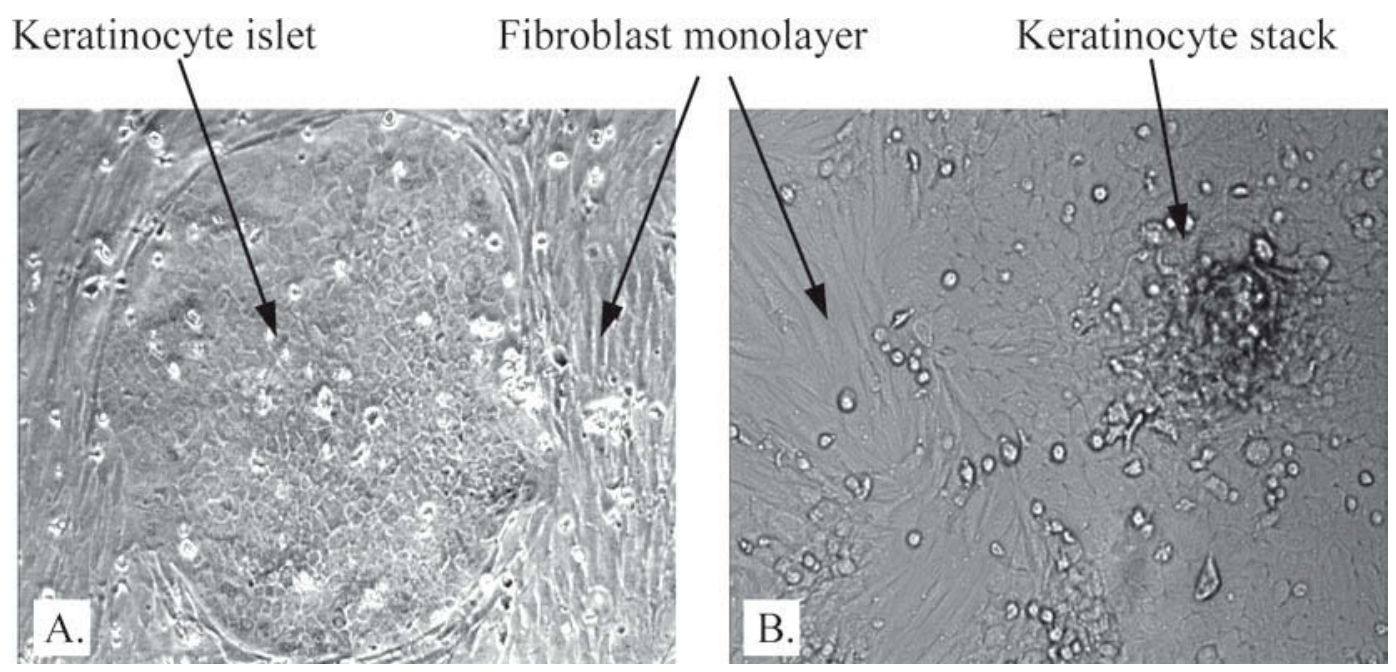

Figure 2. Keratinocyte-fibroblast cocultures 7 d postisolation. Coronary region keratinocytes were overlaid onto 80 to $90 \%$ confluent monolayers of dermal fibroblast in Eagle's minimum essential medium supplemented with $10.0 \%$ fetal bovine serum. Keratinocytes appeared as islets of cobblestone-shaped cells surrounded by swirls of elongated, stellate-appearing fibroblast monolayers walling off a keratinocyte islet (panel A; $100 \times$ magnification). Islets of keratinocytes also formed stacks of cobblestone keratinocytes (panel B; 40× magnification). Pictures represent culture results from 1 of 40 cell preparations.
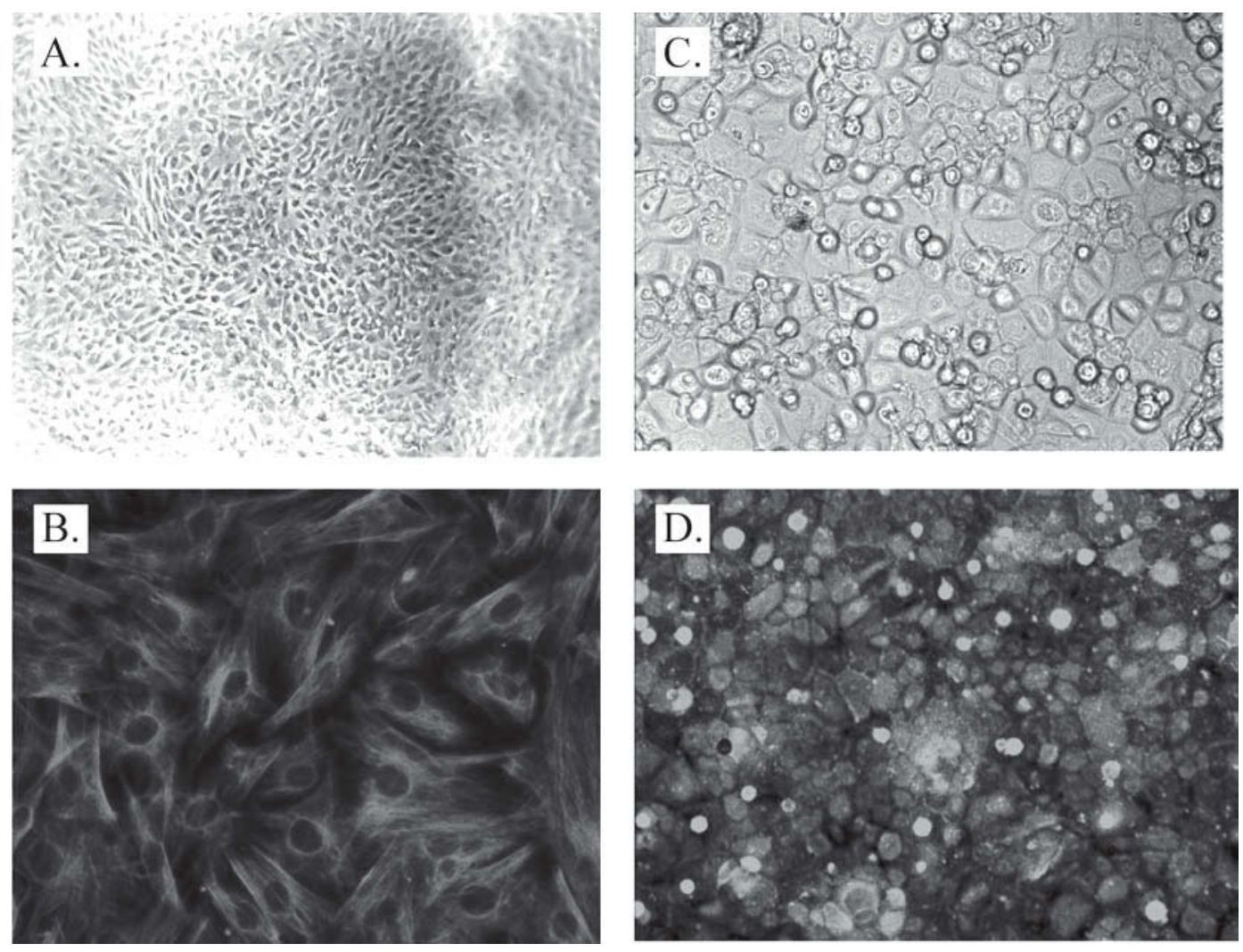

Figure 3. Cell identification with vimentin and cytokeratin immunofluorescent staining. Fibroblasts (panel A; 40× magnification) were identified by the cell marker vimentin using Cy3-conjugated murine anti-vimentin. Keratinocytes (panel C; 100× magnification) were identified by expression of keratin with a primary guinea pig anti-bovine fetal hoof keratin and a secondary fluorescein isothiocyanate-conjugated goat anti-guinea pig IgG. Fibroblasts (panel B; 100× magnification) demonstrated a diffuse and linear red fluorescence of the cytoplasm, whereas keratinocytes (panel D; 100× magnification) demonstrated a green fluorescence diffusely distributed throughout the cytoplasm. Pictures represent culture results from 1 of 40 cell preparations. 

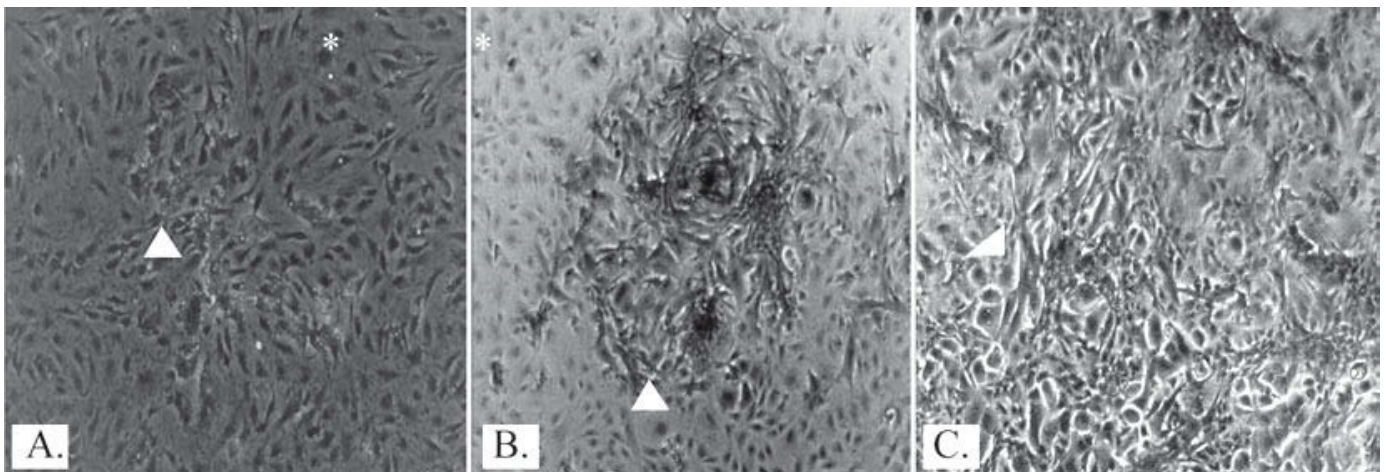

Figure 4. Coronary region keratinocyte colony appearance in keratinocyte-fibroblast coculture (40× magnification). Keratinocytes and fibroblasts were plated in coculture conditions at varying cellular inputs (digital pictures recorded clone growth for the plated concentration of 5.0 $\times 10^{3}$ keratinocytes $/ \mathrm{cm}^{2}$ ) at 2,6 , and $10 \mathrm{~d}$ post-incubation. The number and size of colonies present showed a direct correlation to the increase in days, indicating that colony formation was a time-dependent process. At $2 \mathrm{~d}$ post-incubation (panel A), a confluent monolayer of fibroblasts (*) underlying small colonies of keratinocytes (arrow) was observed. At 6 d post-incubation (panel B), colonies of multiple keratinocytes increased in size (arrow) and became walled off by fibroblasts $(*)$. At $10 \mathrm{~d}$ post-incubation (panel C), larger colonies of stratified keratinocytes (arrow) aggregated on the plate with evidence of cellular stacks above a basal cell layer. Pictures represent results of 1 of 5 experiments performed in triplicate.

at the 2 greater seeding densities, d 10 colony counts were no different from those at 2 and $6 \mathrm{~d}$ (Figure 5B; $P<0.05)$.

The efficiency of colony formation by primary and second-passage coronary region keratinocytes provided an estimate of the proportion of colony-forming abilities within the total keratinocyte population. In as much as colony numbers peaked $6 \mathrm{~d}$ after coculture (Figure $5 \mathrm{~A}$ and $\mathrm{B}$ ), plating efficiency was estimated from $\mathrm{d} 6$ colony numbers across all 3 plating densities. Primary keratinocyte plating efficiency was $1.09 \%( \pm 0.16)$, $1.77 \%( \pm 0.28)$, and $1.38 \%( \pm 0.23)$ at plating densities of $5 \times 10^{3}, 7.5 \times 10^{3}$, and $1.0 \times 10^{3}$ primary keratinocytes $/ \mathrm{cm}^{2}$, respectively. Second-passage keratinocyte plating efficiency was $1.58 \%( \pm 0.71), 1.57 \%( \pm 0.70)$, and $1.31 \%( \pm 0.29)$ at plating densities of $5 \times 10^{3}, 7.5$ $\times 10^{3}$, and $1.0 \times 10^{3}$ primary keratinocytes $/ \mathrm{cm}^{2}$. The efficiency of colony formation was unaffected by keratinocyte plating density within primary $(P<0.05)$ or second-passage keratinocytes $(P<0.05)$.

\section{Fibroblasts Are Necessary and Sufficient for Keratinocyte Colony Formation}

Fibroblasts affected coronary region keratinocyte colony formation $(P<0.05, \mathrm{n}=4)$. Keratinocytes cocultured on fibroblast monolayers produced $144.0 \pm$ 31.6 and $183.0 \pm 53.0$ keratinocyte colonies, respectively, whereas keratinocytes cultured in the absence of fibroblast monolayers produced $0.0 \pm 0.0$ colonies. In the absence of fibroblasts, the keratinocytes appeared as nonadherent, sparse populations of small, rounded, floating cells.

\section{Cytokines and Growth Factors Are Expressed in Keratinocyte-Fibroblast Cocultures}

Quantitative real-time PCR demonstrated that IL$1 \alpha$ mRNA expression was constant until $24 \mathrm{~h}$ and then increased to peak at $144 \mathrm{~h}$ in culture (Figure 6A; $P<$ 0.05). Expression of mRNA for IL-1 receptor type I (IL1-RTI) and IL-1 receptor antagonist (IL1RA) paralleled IL-1 $\alpha$ expression (Figure $6 \mathrm{~A} ; P<0.05$ ). In contrast, IL-1 $\beta$ expression decreased across the 6 -d period (Figure $6 \mathrm{~A} ; P<0.05$ ). The expression of IL1 receptor type II (IL1-RTII) mRNA peaked at $24 \mathrm{~h}$, and slowly declined through $144 \mathrm{~h}$ of culture (Figure $6 \mathrm{~A} ; P<0.05$ ). Early granulocyte-macrophage colony stimulating factor (GM-CSF) mRNA expression $(0,12$, and $24 \mathrm{~h})$ was minimal, but increased from 24 to $144 \mathrm{~h}$ in culture (Figure 6B; $P<0.05$ ). Basal keratinocyte growth factor (KGF) mRNA concentrations increased throughout $144 \mathrm{~h}$ of keratinocyte-fibroblast coculture (Figure $6 \mathrm{~B} ; P<0.05)$. Expression of KGF receptor (KGFR) was not affected until $144 \mathrm{~h}$ in coculture when expression was greater than at earlier time points (Figure 6B; $P<0.05)$. Expression of transforming growth factor $\beta$ (TGF- $\beta$ ) mRNA increased across the entire $144 \mathrm{~h}$ (Figure $6 \mathrm{C} ; P<0.05$ ) of culture, whereas expression of tumor necrosis factor $\alpha$ ( TNF- $\alpha)$ increased early (12, 24, and 48 h) but decreased thereafter (144 h; Figure $6 \mathrm{C} ; P<0.05)$.

\section{DISCUSSION}

Tissue homeostasis in stratified epithelium of the integument and derivatives such as the bovine claw arises through regulated balance between basal cell 

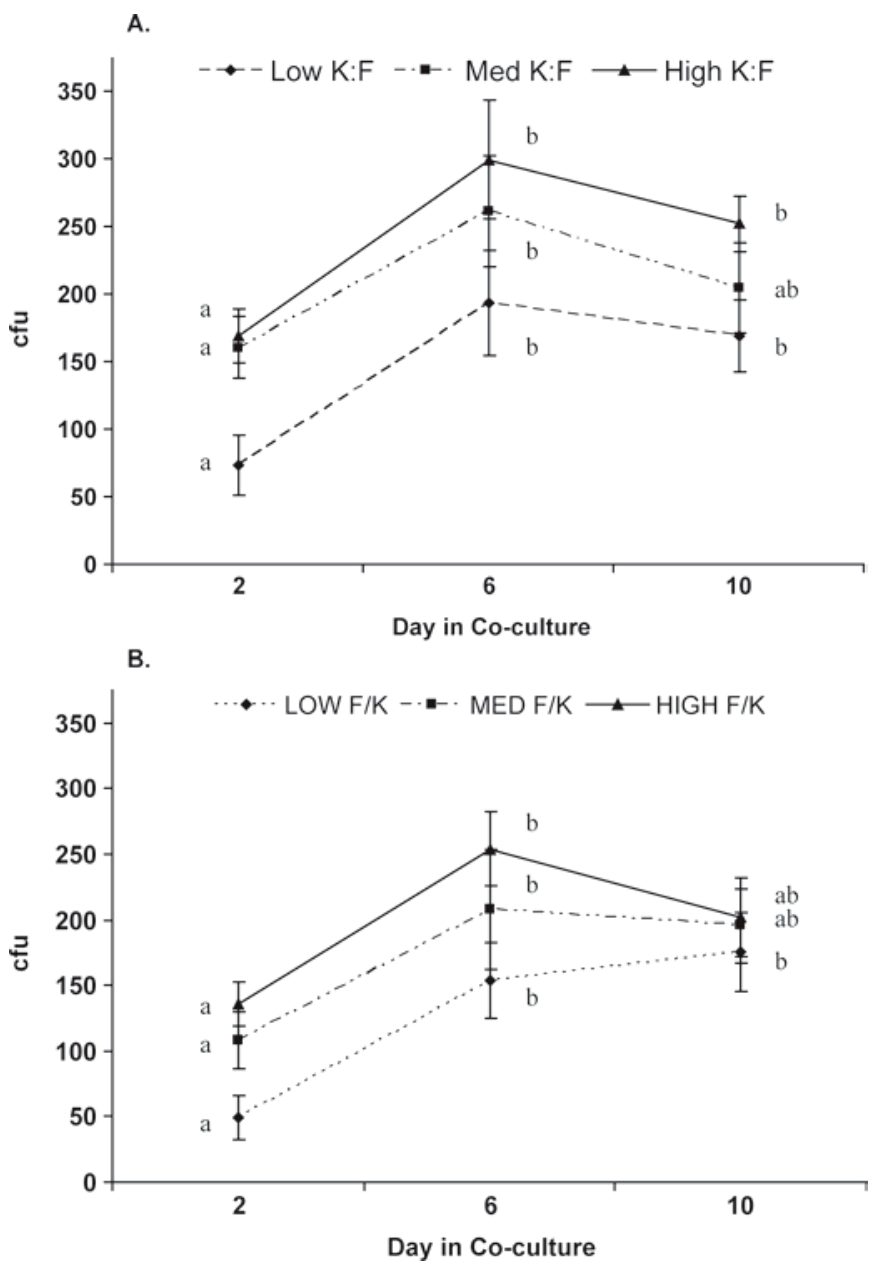

Figure 5. Colony unit formation (cfu) by primary (panel A) and second-passage (panel B) coronary region keratinocytes after 2, 6, and $10 \mathrm{~d}$ in coculture. Epidermal keratinocytes $(\mathrm{K})$ isolated directly from coronary region epidermis or after the second passage in vitro were seeded onto confluent monolayers of dermal fibroblasts $(\mathrm{F})$ at $5.0 \times$ $10^{3}\left(\right.$ low K:F), $7.5 \times 10^{3}\left(\right.$ med K:F), and $1.0 \times 10^{4}$ (high K:F) cells/ $\mathrm{cm}^{2}$. ${ }^{\mathrm{a}, \mathrm{b}}$ Letters represent differences $(P<0.05)$ within cell density across time. Data presented as mean \pm SEM for 5 experiments performed in triplicate.

proliferation and suprabasal cell differentiation. During the process, cells of the innermost basal cell layer enter the cell cycle to generate progeny cells that translocate to the suprabasal layers. Growth activity in suprabasal cells becomes progressively restricted with the onset of programs of differentiation until the cells permanently abort the cell cycle. Terminally differentiated cells of the suprabasal layers of epithelium no longer grow but differentiate into heavily keratinized, cornified cells of the outermost suprabasal layers. Three functional types of clonal activity have been associated with these stages of keratinocyte development (Barrandon and Green, 1987). Holoclones are colonies formed by cells from the basal layer that retain colony-forming activities in-
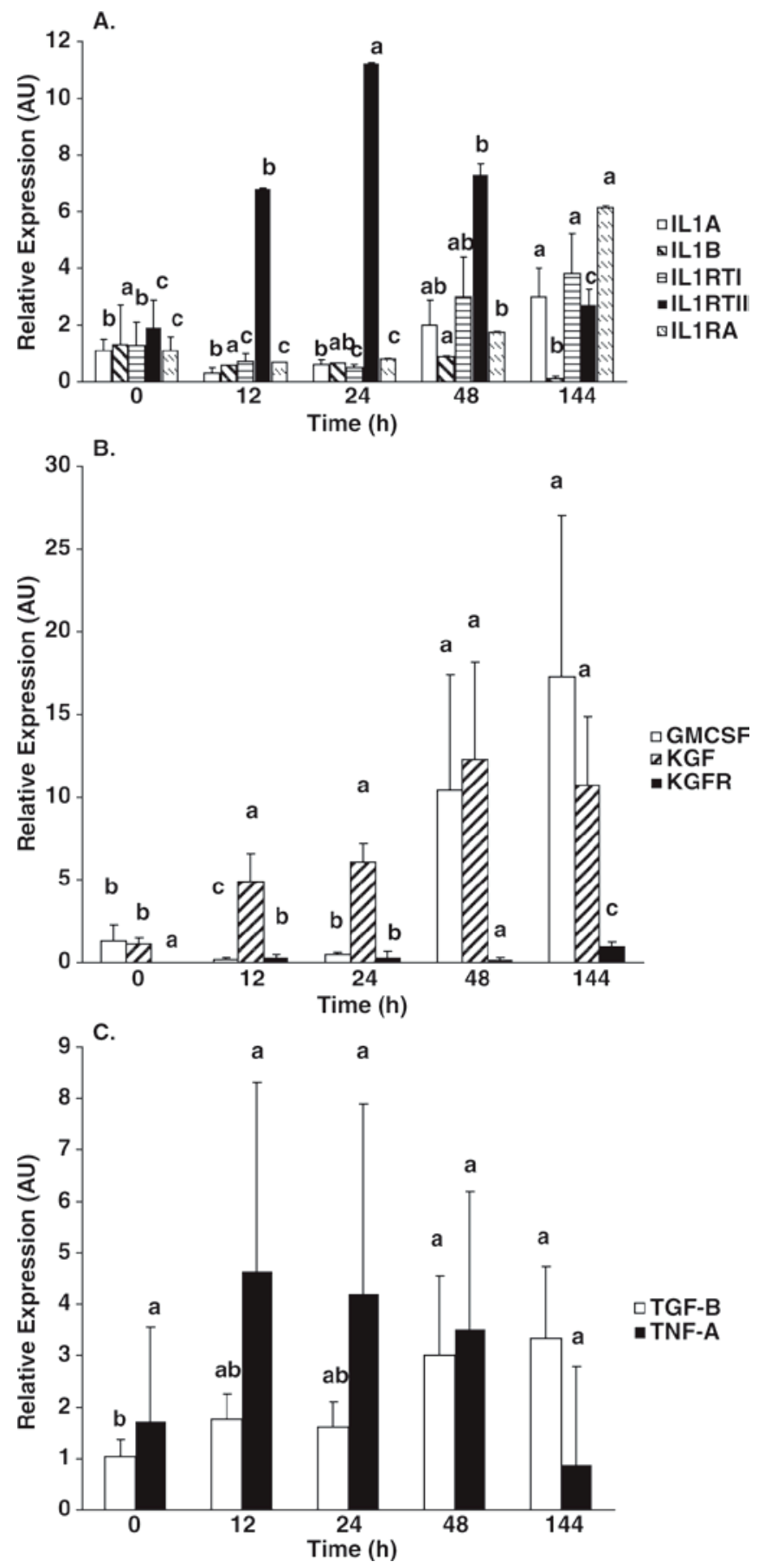

Figure 6. Expression of IL-1 $\alpha$, IL-1 $1 \beta$, IL-1 receptor type I (IL1RTI), IL-I receptor type II (IL-1RTII), and IL-1 receptor antagonist (IL-1RA) mRNA (panel A); granulocyte-macrophage colony stimulating factor (GM-CSF), keratinocyte growth factor (KGF), and KGF receptor (KGFR) mRNA (panel B); and transforming growth factor $\beta$ (TGF- $\beta$ ) and tumor necrosis factor $\alpha$ (TNF- $\alpha$ ) mRNA (panel C) in keratinocyte-fibroblast coculture. Cocultures of coronary region keratinocytes and embryonic fibroblasts were cocultured for 12, 24, 48, and $144 \mathrm{~h}$. Concentrations of mRNA were determined by real-time PCR, normalized against ubiquitin, and converted to relative expression as arbitrary units (AU) compared with time 0. Data are presented as mean \pm SEM of 4 experiments performed in duplicate. ${ }^{\text {acc }}$ Letters represent significance $(P<0.05)$ across time within gene. 
definitely because they do not differentiate. Paraclones and meroclones are colonies formed by cells from the suprabasal layers that transiently retain cell-cycle and colony-forming activities but have also initiated programs of terminal differentiation. These so-called transit amplifying cells eventually abort cell-cycle activities as they progress deeper into the programs of terminal keratinocyte differentiation.

The p63 protein, a member of the p53 family of tumor suppressor transcription factors, orchestrates a balance between mitotic quiescence and entry into the cell cycle (Jensen and Watt, 2006; Senoo et al., 2007). Expression of p63 is high in cells generating holoclones and low to absent in cells that generate paraclones and meroclones (Pellegrini et al., 2001). The p63 protein therefore serves as a marker of cells retaining a high proliferative potential even though p63 does not affect proliferation, lineage commitment, differentiation, or maturation per se (Senoo et al., 2007). In as much as p63-positive cells have been associated with proliferative potential in basal keratinocytes (Parsa et al., 1999; Pellegrini et al., 2001; Yi et al., 2008), we hypothesized that p63 would be most heavily expressed in the basal and first few suprabasal epithelial layers of the bovine coronary region in accordance with results from other species ((Mills et al., 1999; Parsa et al., 1999). Indeed, transverse and longitudinal profiles of normal dermal papilla showed the greatest amount of p63 expression within basal keratinocytes adjacent to the basement membrane of the normal coronary region. The restricted distribution of p63 to nuclei of the basal cells of the coronary region epidermis is consistent with its distribution in the skin (Yang et al., 1998). The intensity of the p63 marker diminished considerably in the suprabasal layers immediately above the basal cells consistent with distributions in human skin (Parsa et al., 1999) and the loss of p63 expression in differentiating epithelial cells (Ghazizadeh and Taichman, 2005; Senoo et al., 2007). The uneven pattern of p63-positive cells across the apex and recesses of the dermal papilla was unexpected but indicated that p63-positive basal cell density was greatest in the intertubular forming epidermis. The significance of this distribution was unclear but was remarkably similar to the distribution of other markers of basal cells $\left(\alpha_{2} \beta_{1}\right)$ in the tips of the deep rete ridges of the skin of the human palm (Jones and Watt, 1993). The uneven, patch-like distribution of p63 among basal cells of the coronary region epithelium was similar to that reported by Parsa et al. (1999) and consistent with the spatially distinct distribution of epidermal basal cells and their overlying progeny that comprise epidermal proliferative units described by Ghazizadeh and Taichman (2001). Assuming p63 expression in the epidermis of the bovine coronary region was in accord with p63 expression in murine and human holoclone cells, our data indicated that holoclone cell distribution dominated the basal layers and was diminished in the suprabasal layers of the coronary region epidermis. Moreover, p63 distribution also indicated that holoclone activity may dominate intertubular compared with tubular areas of the coronary region epidermis.

We developed a coculture system that demonstrated the clonogenic capacity of keratinocytes from the bovine coronary region. Keratinocyte colony formation was time-dependent although the factors supporting growth were not identified. Replicative function in coronary region keratinocytes was observed in a relatively low proportion of unfractionated primary and second-passage keratinocyte populations. Note our estimates were generated from peak colony-forming unit counts (d 6) because d 2 and d 10 counts may have underestimated colony-forming unit efficiencies. Colony-forming unit numbers at d 2 were low most likely because colony formation was in the earliest stages of development and therefore incomplete. At d 10, we noted that rapidly growing colonies appeared to expand and fuse with neighboring colonies, thereby artificially reducing the total colony counts. In as much as the proportion of colony-forming cell numbers in any given population should not change across population size, the absence of an effect of plating density on keratinocyte colonyforming cell estimates within unfractionated primary or the second-passage keratinocyte populations was anticipated. Our results were also consistent with estimated frequencies of replicative cell function in human skin, but greater than the efficiency recorded in porcine skin (Rheinwald and Green, 1975; Hengge et al., 1996). Prevalence estimates may be dependent on technique in as much as estimates as high as 8 to $10 \%$ keratinocyte stem cells in epidermal cell populations have been reported using genetic or cell-surface markers $(\alpha 6$ integrin and CD71; Ghazizadeh and Taichman, 2005).

The functional demonstration of colony-forming unit activity within populations of coronary region keratinocytes provided functional evidence that 1 to $2 \%$ of the epidermal keratinocytes of the coronary region were endowed with cell-cycle activity. We note that colonyforming activity represents total cell-cycle activity that could be distributed across holoclones, paraclones, and meroclones within the coronary region of the claw (Jones and Watt, 1993). We predict that part of this colony-forming unit activity will be sustained indefinitely, whereas another portion of the colony-forming unit activity will terminate as programs of differentiation initiated within the claw progress to completion. Future assessment of holoclone, meroclone, and paraclone numbers will require demonstration of long-term sustainable colony-forming unit function (Rheinwald 
and Green, 1975) coupled with characterization of expression of markers such as Lrig1, p63, $\beta 1$ integrin, $\alpha 6$ integrin, and Delta1, or Cd71, desmoglein 3, and epidermal growth factor receptor (EGFR1; Pellegrini et al., 2001; Ghazizadeh and Taichman, 2005; Jensen and Watt, 2006). The demonstration that colony-forming abilities were retained through second-passage coronary region keratinocytes was consistent with existence of holoclone, paraclone, and meroclone activity within the epithelium of the coronary region.

Data provided herein indicated that dermal fibroblasts of the coronary region were necessary and sufficient to drive growth of coronary region keratinocytes. Keratinocyte colony-forming units developed only in the presence of the dermal fibroblasts. The fibroblast requirement was similar to that established for human and murine keratinocytes in that dermal microvascular endothelial cell or dermal fibroblasts were necessary to support keratinocyte growth (Smola et al., 1993). The bovine results were consistent with fibroblasts serving as a source of growth factors and other cytokines that orchestrate keratinocyte growth and development (Szabowski et al., 2000; Werner and Smola, 2001; Werner et al., 2007) reported distinct increases in mitogenic molecules such as IL-1 $\alpha$, GMCSF, and KGF was associated with increased bromodeoxyuridine uptake during a 6-d period in murine keratinocytes. Nelson and Bissell (2006) showed that epithelial-fibroblast cocultures resulted in production of growth factors, matrix components, and cell communications necessary to trigger keratinocyte growth.

Earlier work in human and murine systems demonstrated a role for IL-1, GM-CSF, and KGF in keratinocyte homeostasis (Werner and Smola, 2001; Werner et al., 2007). Analysis of different cellular compartments in murine and human models revealed that KGF and GM-CSF expressed by fibroblasts stimulated expression of IL-1 in overlying keratinocytes. Interleukin-1, in turn, induced more growth factor (KGF and GM-CSF) expression in the underlying fibroblast compartment (Smola et al., 1993; Werner and Smola, 2001). The bovine data were in accord with these findings because increased IL-1 $\alpha$ and IL1RTI receptor expression paralleled increased GM-CSF and KGF expression. Results from the bovine coculture support a model in which coronary region keratinocytes interacted with dermal fibroblasts and mutually coinduced IL-1, KGF, and GM-CSF. Coinduction of these factors in association with keratinocyte colony formation is consistent with results from human and murine keratinocyte data, whereby either IL-1 $\alpha$, IL-1 $\beta$, or both triggered increased expression of KGF and GMCSF, which stimulated keratinocyte colony formation in bovine cocultures.
Bovine transcriptional data also indicated modification of the IL-1 signals; therefore, growth factor production in the bovine system could be altered by changes in IL-1RTI, IL-1RTII, and IL-1RA expression. Conceivably, IL-1 signals for KGF and GMCSF expression in bovine cultures could be diminished by high concentrations of IL-1RTII IL-1RA relative to IL$1 \alpha$, IL-1 $\beta$, and IL-1RTI. A balance of all these factors could coregulate IL-1 and secondarily alter activities of KGF, GM-CSF, or both in the bovine coculture system (Kupper and Groves, 1995; Groves et al., 1996; Murphy et al., 2000). The effect of TNF- $\alpha$ in regulating keratinocyte proliferation and homeostasis was unclear even though TNF- $\alpha$-dependent nuclear factor $\kappa \mathrm{B}$ activation and upregulation of multiple proinflammatory cytokines and mitogens should potentiate keratinocyte proliferation (Banno et al., 2005).

The transcriptional data from the bovine in vitro model may not precisely depict all the cell-to-cell interactions occurring in the normal bovine claw. For example, serum, a constituent in these coculture systems has been shown to induce genes associated with fibroblast proliferation, signal transduction, tissue remodeling, homeostasis, and inflammation (Smola et al., 1993; Iyer et al., 1999; Szabowski et al., 2000). In fact, gene expression in serum-stimulated fibroblasts has been proposed to resemble profiles associated with wound healing rather than normal tissue homeostasis (Iyer et al., 1999). Elevated transcription of TGF $\beta$ in these bovine cocultures may reflect wound healing responses in as much as TGF $\beta$ orchestrates a coordinated sequence of disassembly of desmosome and cell adhesions with actin filament and matrix protein remodeling typical of healing responses in keratinocytes. Clearly, more work is required to determine the relationship between cytokine and growth factor expression reported in these bovine cocultures and cell-to-cell interactions in normal or injured epidermal and dermal structures of the claw; however, the ability of fibroblast feeders in only $0.1 \%$ FBS to enhance keratinocyte development indicated that the effects observed herein were more indicative of a specific cell-to-cell interaction than of serum-driven events. Conceivably, events disturbing the supportive role(s) of dermal fibroblasts in keratinocyte homeostasis contribute to the biochemical (Hendry et al., 2003) and morphologic (Budras et al., 1996) evidence of altered keratinocyte activity during poor-quality claw horn production.

\section{REFERENCES}

Banno, T., A. Gazel, and M. Blumenberg. 2005. Pathway-specific profiling identifies the NF-kappa B-dependent tumor necrosis factor alpha-regulated genes in epidermal keratinocytes. J. Biol. Chem. 280:18973-18980. 
Barrandon, Y., and H. Green. 1987. Three clonal types of keratinocyte with different capacities for multiplication. Proc. Natl. Acad. Sci. USA 84:2302-2306.

Budras, K. D., C. Mulling, and A. Horowitz. 1996. Rate of keratinization of the wall segment of the hoof and its relation to width and structure of the zona alba (white line) with respect to claw disease in cattle. Am. J. Vet. Res. 57:444-455.

Ghazizadeh, S., and L. B. Taichman. 2001. Multiple classes of stem cells in cutaneous epithelium: A lineage analysis of adult mouse skin. EMBO J. 20:1215-1222.

Ghazizadeh, S., and L. B. Taichman. 2005. Organization of stem cells and their progeny in human epidermis. J. Invest. Dermatol. 124:367-372.

Groves, R. W., T. Rauschmayr, K. Nakamura, S. Sarkar, I. R. Williams, and T. S. Kupper. 1996. Inflammatory and hyperproliferative skin disease in mice that express elevated amounts of the IL-1 receptor (type I) on epidermal keratinocytes: Evidence that IL-1-inducible secondary cytokines produced by keratinocytes in vivo can cause skin disease. J. Clin. Invest. 98:336-344.

Hendry, K. A., C. H. Knight, H. Galbraith, and C. J. Wilde. 2003 Basement membrane integrity and keratinization in healthy and ulcerated bovine hoof tissues. J. Dairy Res. 70:19-27.

Hengge, U. R., E. F. Chan, V. Hampshire, R. A. Foster, and J. C. Vogel. 1996. The derivation and characterization of pig keratinocyte cell lines that retain the ability to differentiate. J. Invest. Dermatol. 106:287-293.

Iyer, V. R., M. B. Eisen, D. T. Ross, G. Schuler, T. Moore, J. C. Lee, J. M. Trent, L. M. Staudt, J. Hudson Jr., M. S. Boguski, D. Lashkari, D. Shalon, D. Botstein, and P. O. Brown. 1999. The transcriptional program in the response of human fibroblasts to serum. Science 283:83-87.

Jensen, K. B., and F. M. Watt. 2006. Single-cell expression profiling of human epidermal stem and transit-amplifying cells: Lrig1 is a regulator of stem cell quiescence. Proc. Natl. Acad. Sci. USA 103:11958-11963.

Jones, P. H., and F. M. Watt. 1993. Separation of human epidermal stem cells from transit amplifying cells on the basis of differences in integrin function and expression. Cell 73:713-724.

Kupper, T. S., and R. W. Groves. 1995. The interleukin-1 axis and cutaneous inflammation. J. Invest. Dermatol. 105:62S-66S.

Livak, K. J., and T. D. Schmittgen. 2001. Analysis of relative gene expression data using real-time quantitative PCR and the 2(-delta delta $\mathrm{C}(\mathrm{T})$ ) method. Methods 25:402-408.

Maas-Szabowski, N., A. Shimotoyodome, and N. E. Fusenig. 1999. Keratinocyte growth regulation in fibroblast cocultures via a double paracrine mechanism. J. Cell Sci. 112:1843-1853.

Mills, A. A., B. Zheng, X. J. Wang, H. Vogel, D. R. Roop, and A Bradley. 1999. P63 is a P53 homologue required for limb and epidermal morphogenesis. Nature 398:708-713.

Mulling, C. K., H. H. Bragulla, S. Reese, K. D. Budras, and W. Steinberg. 1999. How structures in bovine hoof epidermis are influenced by nutritional factors. Anat. Histol. Embryol. 28:103108.

Murphy, J. E., C. Robert, and T. S. Kupper. 2000. Interleukin-1 and cutaneous inflammation: A crucial link between innate and acquired immunity. J. Invest. Dermatol. 114:602-608.

Murray, R. D., D. Y. Downham, M. J. Clarkson, W. B. Faull, J. W Hughes, F. J. Manson, J. B. Merritt, W. B. Russell, J. E. Sutherst, and W. R. Ward. 1996. Epidemiology of lameness in dairy cattle: Description and analysis of foot lesions. Vet. Rec. 138:586-591.

Nelson, C. M., and M. J. Bissell. 2006. Of extracellular matrix, scaffolds, and signaling: Tissue architecture regulates development, homeostasis, and cancer. Annu. Rev. Cell Dev. Biol. 22:287-309.

Parsa, R., A. Yang, F. McKeon, and H. Green. 1999. Association of p63 with proliferative potential in normal and neoplastic human keratinocytes. J. Invest. Dermatol. 113:1099-1105.

Pellegrini, G., E. Dellambra, O. Golisano, E. Martinelli, I. Fantozzi, S. Bondanza, D. Ponzin, F. McKeon, and M. De Luca. 2001. p63 identifies keratinocyte stem cells. Proc. Natl. Acad. Sci. USA 98:3156-3161.

Rheinwald, J. G. and H. Green. 1975. Serial cultivation of strains of human epidermal keratinocytes: The formation of keratinizing colonies from single cells. Cell 6:331-343.

Senoo, M., F. Pinto, C. P. Crum, and F. McKeon. 2007. p63 is essential for the proliferative potential of stem cells in stratified epithelia. Cell 129:523-536.

Smola, H., G. Thiekotter, and N. E. Fusenig. 1993. Mutual induction of growth factor gene expression by epidermal-dermal cell interaction. J. Cell Biol. 122:417-429.

Szabowski, A., N. Maas-Szabowski, S. Andrecht, A. Kolbus, M. Schorpp-Kistner, N. E. Fusenig, and P. Angel. 2000. c-jun and JunB antagonistically control cytokine-regulated mesenchymalepidermal interaction in skin. Cell 103:745-755.

Tomlinson, D. J., C. H. Mulling, and T. M. Fakler. 2004. Invited review: Formation of keratins in the bovine claw: Roles of hormones, minerals, and vitamins in functional claw integrity. J. Dairy Sci. 87:797-809.

Werner, S., T. Krieg, and H. Smola. 2007. Keratinocyte-fibroblast interactions in wound healing. J. Invest. Dermatol. 127:998 1008 .

Werner, S., and H. Smola. 2001. Paracrine regulation of keratinocyte proliferation and differentiation. Trends Cell Biol. 11:143-146.

Yang, A., M. Kaghad, Y. Wang, E. Gillett, M. D. Fleming, V. Dotsch, N. C. Andrews, D. Caput, and F. McKeon. 1998. P63, a P53 homolog at 3q27-29, encodes multiple products with transactivating, death-inducing, and dominant-negative activities. Mol. Cell 2:305-316.

Yi, R., M. N. Poy, M. Stoffel, and E. Fuchs. 2008. A skin microRNA promotes differentiation by repressing "stemness". Nature $452: 225-229$. 